\title{
XXVI. On the theory of explosions
}

\section{Richard Threlfall B.A.}

To cite this article: Richard Threlfall B.A. (1886) XXVI. On the theory of explosions, Philosophical Magazine Series 5, 21:130, 165-179, DOI: 10.1080/14786448608627832

To link to this article: http://dx.doi.org/10.1080/14786448608627832

曲 Published online: 29 Apr 2009.

Submit your article to this journal

Џ Article views: 3

Q View related articles $\asymp$ 
LONDON, EDINBURGH, AND DUBLIN

\title{
PHILOSOPHICAL MAGAZINE
}

\author{
AND \\ JOURNAL OF SCIENCE.
}

[FIFTH SERIES.]

$M A R C H 1886$.

XXVI. On the Theory of Explosions. By RICHARD Threlfall, B.A., Assistant Demonstrator of Physics in the Cavendish Laboratory, Cambridge*.

$I^{N}$ $\mathrm{N}$ his papers on the action of Detonators, Sir Frederick Abel described some curious experiments, which he strove to account for by a hypothesis of "synchronous vibrations." This hypothesis has been treated at considerable length by Berthelot in his work Sur la Force des Matières Explosives; and although much light, both experimental and theoretical, has been thrown on the matter by Vieille and Berthelot, the explanations offered by the latter do not seem altogether satisfactory. I have therefore been led to imagine that perhaps something of interest might be gathered from a study of the behaviour of the products of explosion, especially as regards the manner in which they escape from the centre at which the explosion takes place.

Much might possibly be learned from a measurement of the velocity of transmission of a shock to points at small distances from the centre of explosion. This would be merely a question of apparatus. Lord Rayleigh has suggested to me the use of a sensitive flame and revolving mirror, which would at all events give some idea of the sort of disturbance experienced. But the best method of all seems to me to begin by examining cases where the results of explosion can be seen; and for this purpose I carried out the following series of experiments during last autumn.

* Communicated by the Author.

Phil. Mag. S. 5. Vol. 21. No. 130. March 1886. N 
A tank, measuring a yard each way, was constructed and filled with windows of strong plate glass about a foot square in each of two of its adjacent sides. This tank was filled with water. It was at first intended to introduce small charges of fulminate of mercury contained in glass bulbs of $\frac{1}{2}$ inch in diameter, and capable of being fired electrically. The bulbs were to be surrounded by water coloured by some dye-stuff, and the distribution of this after the explosion was to be observed. However, this was soon found to be unnecessary, for the débris of explosion afforded sufficient guide. One observer (Mr. William Walker) was generally stationed at one of the windows; I fired the charge by means of a contact-key in connection with a small coil, and looked in from the top, or side, as seemed most desirable. Wo soon found that the general effects were pretty regular in some ways, and irregular in others. When a small glass globe one half-inch in diameter had been filled with pure fulminate of mercury shaken well down, but not pressed, the ends of a pair of insulated wires twisted together were passed in and pushed down to measurement till the points were as nearly as possible about the middle of the globe. A little melted paraffin was run round the neck of the bulb to make it water-tight, and the whole was lowered by means of the wires to a constant depth of about 18 inches. At first we were astonished to find that the débris of explosion had the appearance of being shot down to the bottom; not in a jet, but with exactly the rolling motion that smoke has in coming out of a chimney, -as if, in fact, there was vortex motion of some sort. I was anxious to try instantaneous illumination, but found I had not the apparatus to produce a bright enough spark. I shall hope, however, to be enabled to do this at some future time. Noticing the constancy of the downward action of the explosion, it occurred to me that it was produced by the want of symmetry introduced by the neck and wires of the cartridge. In order to test this I turned the next bulb on its side, and then the débris seemed to move with its peculiar rolling motion away from the neck. In fact, the appearance presented to the unaided eye was that of a more or less definite column of rolling white smoke shot out with great velocity, and coming to rest very rapidly when about tive inches from the centre, as if acted on by an irresistible force. I also made some experiments by exploding a charge in the centre of a Florence oil-flask filled with red dye and immersed in the water. The dye was shot out with the débris, and the flash appeared to be suddenly stopped some two or three inches outside where 
the flask would have been if it had not disappeared. There were so many sources of misinterpretation to be feared in this mothod that I did not continue it, but noted the peculiar rolling, and the dead-beat motion, of the dye as it was shot out.

Unfortunately, just as I had reached the conclusion of these observations, one of the windows gave way, and I decided to board them both up, and take some observations with an impact gauge or ganges, which I had meanwhile constructed. Each gauge consisted of a short holiow cylinder of brass, about 3 inches in diameter and an inch deep. One end was made of stout brass, and the other had a sheet of india-rubber stretched tambourine-fashion over it. A brass tube, $\frac{1}{2}$ inch in diameter and 18 inches long, was fistened into the cylinder in such a way that when the plane of the india-rubber was vertical, the tube was also vertical. Into this brass tube there projected and was cemented a tube of glass projecting upwards about a meter, and having an internal diameter of about $\frac{\lambda}{16}$ in. Two such gauges wero made, and carefully fastened into, and backed by, wooden supports, on which were carried the graduated seales by whose aid any rise or fall of liquid in the glass tube conld be measured. These supports were then firmly fastened to the two adjacent sides of the tank. The centres of the india-rubber faces were as nearly as possible in the same horizontal plane, and about 18 inches below water-level when the tank was full. The gauges themselves were filled with water (coloured with rosaniline) to such a point that the coloured liquid stood about an inch above water-level when the tank was full. Precautions were of course taken to prevent any air being entrapped in the gauges. The explosions were caused entirely by fulminate of mercury enclosed in glass bulbs of about $\frac{1}{4}$ inch in diameter, and blown as carefully as $I$ was able. The covered wires projected their bare ends pretty accurately to the middle of the bulbs, and these latter were rendered water-tight by passing round the joints a little melted paraffin.

A wooden rod laid across the top of the tank had an iron rod projecting from it vertically downwards to a depth of about 16 inches. The wires carrying the charge were tied to this rod, and allowed to project beyond its end sufficiently far to place the bulb in the horizontal plane of the centre of the two gauges.

By means of marks on the sides of the tank and of the wooden rod, the latter could easily be adjusted so as to place the bulb at a point equidistant from the two gauges. This distance was varied in different experiments by shifting the 
gauges and the wooden bar supporting the charge. The gauges were always equidistant from the explosion centre.

A key placed in a convenient position enabled me to fire the charge whenever I desired, without distracting my attention from the reading of one of the gauges. The other gauge was read by Mr. Walker, who soon learned the way of it and became an accurate observer. The results were at first surprising. It will be noticed that extreme precautions were taken to make the distribution of effect about the vertical axis through the point of ignition as symmetrical as possible. In spite of these precautions I found that the gauges by no means kept pace with each other. First one would give a large reading, and then the other. The average reading was about 3 inches of coloured water. I finally traced this out to the almost unavoidable nnevenness in the glass of the bulbs. A new set of bulbs were therefore blown of thinner glass, and carefully examined as to uniformity before being used. The readings now became more consistent, though not so much so as I should like to have seen them. While considering how to make certain of a more perfect symmetry, I discovered a peculiar action which finally caused me to abandon this form of gauge entirely, as I could not be sure about its theory. I had imagined that the energy of explosion would be transmitted by the almost unstrained layer of thin india-rubber without appreciable loss to the interior of the gauge, and that the result of the impact would be a rise of the liquid in the tube, much as if the gauge had been struck on its face by a hammer. Now the tambourine-shaped end of the gauge and about 6 inches of the connecting-tube projected freely into the water below the wooden support, so that the distance from the back of the gauge to the side of the tank was about 4 inches.

After several shots had been fired, 1 noticed that both the tubes of the gauges were bent towards the centre of the explosion, not away from it. A wedge placed behind the tambourine and between it and the side of the tank, and pressed tight in, increased this effect. How it came about, I was at a loss to discover; but the result was that I decided I knew too little about the motion of the water to trust to the proper deformation of the india-rubber. The rapidity with which the coloured liquid rose and fell was also a drawback. The gauges were troublesome to set besides, so that I finally determined to make two new ones. In these I adopted a pendulum method. The two ends of a bit of brass tube, one inch in diameter and four inches long, were closed by caps. Through the centre of these caps I bored holes three eighths of an inch in diameter and immediately opposite one another. The tube 
was fastened horizontally to a wooden support, and a brass rod moved piston-wise through the holes in the ends; the rod was about six inches long. Inside the tube was coiled a spiral spring of hard-brass wire; one end of the spring rested against the inner surface of one of the caps, and the other was pinned to the brass rod. The result was, that the rod was always held by the spring in one position. One end of the rod was furnished with a brass disk about three inches in diameter, the other end abutted on the bob of a lever pendulum so suspended as always to press slightly on it. The pendulum was supported by a small shaft passing through it and through holes in two brass brackets. The distance from the bob (which weighed about six ounces) to the fulcrum was one inch, and the pendulum was extended upwards about 20 inches by a pointer of sheet zine; the end of this pointer moved over a paper seale. When properly supported in the tank the gauges occupied much the same position as the ones previously employed. The flat disks of the gauges were placed so as to face towards the centre of explosion. These gauges seemed to leave little to be desired either as to convenience of reading or otherwise, and, both being exactly alike, gave tolerably consistent readings.

By firing some dozen charges, arranged as symmetrically as possible, I managed to find out that the indications of the gauges were proportional, in about the ratio of 1.3 to 1 . I did not require to be very accurate, because all I was looking for was to find whether the streams of débris formerly observed through the window were also streams of explosive effect.

To decide this I made the explosion cartridges purposely unsymmetrical, either by having the glass too thick on on $\theta$ side, or by turning up the ends of the covered wires so that they entered the bulb horizontally and facing one of the gauges. The effects now became more puzzling, but on the whole there can be no question that the gauge towards which the bulb was turned suffered most. In fact the direction taken by the streams of explosive energy appeared to coincide with the directions of projection of débris, and with the direction foretold from the initial conditions.

The experiments were repeated at various distances and in various manners with more or less compressed charges, and with variations in the position of the firing-point. The pendulum readings were on the whole certainly proportional to the direction of explosion as foretold from the initial conditions. Of course, in some few cases, there were unexpected actions on the gauges; but this was hardly avoidable, since I had seen by the previous experiments how small a change in 
initial conditions could lead to great variations in the result. The position of the firing-point was the least satisfactory part of the experiments ; and it seems probable that when the gauges did not go as they were expected to go, the change of effect was to be traced to imperfect centering of the firingpoint; about 10 per cent. of experiments would decline to travel on the paths laid out for them.

Some considerable difficulty was experienced in making the tank water-tight. The continual shock of explosion stended to produce leaks, and for some time this was very tiresome. Mr. Walker, however, recollected that the introduction of a little horse-manure into the tank was a method sometimes employed for stopping leaks, and on trying it we found it succeeded in absolutely stopping each leak as it was caused within a very few minutes; the fine particles having about the same density as water remain suspended for a long time and get carried into the holes as soon as a leak appears.

These experiments leave very little doubt in my own mind that the direction in which the maximum explosive effect is transmitted will, in a great measure, depend on the initial arrangement of surrounding obstacles; at all events, when the explosion is caused by fulminate of mercury and small charges are used.

The shock of an explosion must be transmitted in one or more of three different ways:-

I. By actual bodily motion of the products of explosion through the surrounding medium, either alone or becoming gradually more and more mixed up with the medium itself, which is thereby also set in motion.

II. By an undulatory motion set up in the medium.

III. B̈y a vortex-ring motion.

In the explosion of gunpowder, and other slow explosives, the energy is doubtless transmitted chiefly by I. and II.

The distance to which a considerable quantity of the energy may be conveyed by means of waves of comparatively great amplitude is in some cases remarkably great. This is evidenced by the effects produced by the explosion of powder magazines. In the case of the fulminates of mercury and silver, gun-cotton and nitro-glycerine, that is explosives of the class examined under water, the effect falls off very rapidly with the distance, and in water, at all events, is of a directed character.

This would point to the third mode of transmission being in these cases of some importance; and if we consider the way in which the products of explosion escape, we shall find that the conditions for the production of vortex motion do in fact exist. Let there be a sphere of fulminate of mercury 
fired from its geometrical centre. Then by Vieille's experiments (Comptes Rendus, 1882 and 1883) on the time of explosion, it seems likely that the outer portions of fulminate will be decomposed before they are removed to any appreciable distance from their original positions. We shall therefore have a sudden expansion in all directions, caused by the increase in volume of the explosive substance during the explosion. There seems no reason under perfectly symmetrical conditions why the expansion should not go on as it began, until the cooling of the sphere of hot gases becomes so marked as to prevent further expansion.

If the conditions, however, are not such as to allow of symmetrical expansion-as always occurs in practice-then we shall have the bounding surface of the explosion-gases more curved in some places than in others-that is, the strain will be greater at some parts than at others, and in fact may become so great at points of great curvature as to lead to what I venture to call a state of "break-down." In other words, the compressed gases will in this case escape, not by gradual expansion but by jets, from points whose position is fixed by the conditions of explosion. In these jets we should have the necessary and sufficient conditions for the establishment of vortex motion. If vortex motion were set up, then it seems likely that much greater effects might be transmitted in some directions than in others, though at considerable distances the effects would tend to become uniform in all directions. This view of the actions of explosions will, I think, enable us to explain several difficulties which I mentioned as occurring in the interpretation of Abel's experiments. These experiments are fully described by Abel in various papers in the 'Philosophical Transactions,' and in the Annales de Chimie et de Physique, vol. 165. They have been discussed at some length by M. Berthelot in his work on Explosives, and though much light has been thrown on many points, I venture to think that the complete elucidation of others is still to be sought. Among these I would place the three following :-

The want of correspondence between the explosive actions, as measured by the effect produced on copper plates, and the effects produced in causing other explosions. The apparent capriciousness of explosions of the more violent kinds; and the production of explosions by influence.

With respect to the explosion of gunpowder, M. Berthelot has nothing new to add to the generally received theory that it differs in nothing from ordinary combustion, except that it is more rapid.

The theory of detonation, however, is more fully treated, 
and is summed up by M. Berthelot somewhat as follows:The kinetic energy of the shock of the explosion (by the detonator) is transformed into beat at the point struck; the temperature of this point is thus raised to the temperature of explosion; a new shock is produced which raises the temperature of the neighbouring portions to the same degree; they then explode and the action is thus propagated with an everincreasing velocity.

Many experiments tend to show the justness of this view. To begin with, Abel found that almost any variety of effect could be obtained by burning explosives under diminished pressure. For the lower the pressure the more easily do the products of decomposition escape, and carry with them the energy due to their liberation. By this means the temperature of explosion is constantly kept down, and the chemical character of the products modified in such a way that they correspond to the temperature. In other words, the compounds liberated are as a rule more complex than those which would be set free at a higher temperature, and therefore the energy run down is less.

A gain, it will come to the same thing, so far as the propagation of an explosion is concerned, whether the products of decomposition are facilitated in their escape by conducting the experiment in a partial vacuum, or whether the decomposition is itself so slow that the products are enabled to escape without marked hindrance under ordinary pressure. Now the resistance of the air to the escape of the products of combustion will depend on the rate at which they are liberated. And the shock given to neighbouring portions of the explosive will be proportional to the pressure of the explosion gases at these points ; and, therefore, ultimately to the resistance of the air, and hence to some function of the velocity of decomposition. But, in order to convert an explosion by combustion into an explosion by detonation, what is required is, that the temperature of any point shall be raised sufficiently to determine its complete, as distinguished from its incomplete, decomposition. The raising of the temperature of any point, however, will depend on the violence of the shock to which it is subjected; and this, as before stated, will be proportional to some function of the velocity of the decomposition producing it If the necessary temperature is anywhere attained, we shall have detonation thereafter; if not, an explosion by combustion will result. It appears, inereiore, that in order to produce a detonation, we require the initial velocity of decomposition to rise above a certain minimum value; that there is in fact a "critical velocity" of 
initial decomposition determining the kind of reaction which ultimately takes place. If the temperature of the whole mass be previously raised, then the "critical" velocity will become less. Berthelot considers that a specific change takes place in the stability of an explosive as its temperature is raised. This is doubtless true; but if a minimum temperature of any part be the necessary and sufficient condition for the production of a detonation, then the ease with which it can be obtained, when the mass starts with a high temperature, will cateris paribus be grenter than if the original temperature is low. If, therefore, we find that nitro-glycerine is more liable to detonation the higher its initial temperature, we shall not be required to make any assumption as to "increas"d sensitiveness," since we see that the minimum temperature will be more easily reached, and that therefore the "critical" velocity of initial decomposition may be smaller. In other words, supposing we try to detonate nitro-glycerine by an explosive which just fails at ordinary temperatures, we should expect its chances of success to increase as the temperature rises; and this does in fact oceur.

The sensitiveness of an explosive to detonation will also, as Berthelot points out, depend on its state of mechanical aggregation. The critical velocity required to produce detonation will ceteris paribus depend on the nature and value of the elastic constants of the explosive as well as of the medium in which it is to be exploded. We should, in fact, expect a change in the critical velocity of detonation if we exchanged the viscous resistance of liquid nitro-glycerine for the elastic resistance of the same substance when frozen. Again, it seems possible, as a result of this theory, that less powerful detonation might be required to explode a given substance in water than in air; I am not, however, aware of any experiments on this point. And so in other cases, though the critical velocity of detonation must necessarily be a very complex function, and difficult to predict, I see no reason on that account to minimise its importance. On the other hand, it seems to me to be in complete harmony with Abel's experiments, and substantially embodies the view set forth by Dixon in his paper "On Conditions of Chemical Change in Gases," Phil. Trans. 1884. As far as I know, there is not a single experiment which offers any evidence against it. What is required by the theory for the formation of a detonation is that a small part of the mass should be raised above a given temperature, and not that a large portion should be raised to a temperature below it.

This leads at once to the consideration of the second point, 
viz. the action of detonators. The apparently anomalous effects discovered by Abel may be summed up by taking the most extreme case. Gun-cotton could be detonated by a charge of fulminate of mercury, whereas ten times as much nitro-glycerine was required to cause a similar sample of guncotton to detonate. By firing the detonating charges on copper plates, Abel naturally observed that the destruction produced by the nitro-glycerine was much the greater, and hence concluded that some other factor, besides the "explosive violence," must come into play. This is undoubtedly true, but the mistake arises in looking at the experiments from one point of view alone, viz. that of the copper plate. There will be no effect produced on the plate at all till the resistance of the air becomes greater than that of the plate; and this will never be the case, however great the volume of gas liberated, unless the time of explosion is sufficiently short. The resistance of the air varies at least as the square of the velocity of attack ; and therefore this will be the conditioning factor of the destructive effect producible by explosions in free air. For a given increment of volume occurring in an explosion till the time of explosion diminishes to a certain value depending on the strength of the plate, no effect will be observed; directly this limit is passed, the destructive effects will depend, in the usual manner, on the quantity of energy liberated. There is in fact a critical velocity of explosion, below which the plate will not be attacked. But in a detonation the case is different. We do not require any great destructive effects, we only require that the time should be so short that a portion, no matter how small, of the substance to be detonated shall be raised to the appropriate temperature. If the detonator has a time of explosion too great, then, although the air may be the stronger obstacle and the explosive be destroyed, no detonation will be produced. This is precisely what happened in Abel's experiments, where the gun-cotton was blown to pieces by the nitro-glycerine. The instantaneous rise of pressure is not so great for nitro-glycerine as for fulminate of mercury, though the energy run down is much greater. This point has been satisfactorily proved by Vieille in his experiments with the crusher-gauge. Moreover the density of mercury fulminate is three times that of nitro-glycerine, which allows a given mass to be on the whole much nearer its work if it consist of fulminate of mercury than if it consists of nitro-glycerine.

We ought not therefore to be surprised that the detonation of gun-cotton is easily accomplished by fulminate of mercury, and hardly accomplished at all by nitro-glycerine. If 
there is any surprise, it would seem more fitting that it should be exhibited at the detonation which large charges of nitro-glycerine seem able to effect.

This fact would tend to show merely that nitro-glycerine has a velocity very near the critical point for gun-cotton, so much so, that when large charges are employed the acceleration in the explosion of the nitro-glycerine is sufficient to pass the limit. We know from Dixon's work on gases that at first the explosion gains in velocity till the steady velocity of detonation is obtained; and the re seems no reason against, but, on the other hand, every probability in favour of, the sanie thing taking place in nitro-glycerine.

Above and beyond this the difference in the mode of application of the two detonators must be taken into account. In Abel's experiment the fulminate was enclosed in a tube of copper or tin plate, while the nitro-glycerine was merely applied in a capsule whose diameter was large compared with its depth. The upper end of the fulminate tube was probably closed by the electric firing apparatus; and this, as was shown by the experiments in water, already described, together with the fact that the fulminate was fired at the top, would give it an enormous advantage. For there is considerable probability that in explosions of high velocity in air, the final mode of "break-down" of the gas liberated is very dependent on the initial conditions, just as $I$ found it to be in water. The nitro-glycerine was deprived by Abel of these advantages; and for these and the reasons above mentioned, though it was able to blow blocks of compressed gun-cotton into powder, and even to cause some of this powder to penetrate the hard wood of the support, it failed to cause detonation.

The other apparently anomalous facts observed by Abel require further treatment, and most of all those explosions by influence which seem, at first sight, only explicable by some theory such as that of synchronous vibrations suggested by Abel himself.

\section{On the Hypothesis of Synchronous Vibrations.}

The difference in the behaviour of nitro-glycerine and fulminate of mercury regarded as detonators, led Abel to suggest that there might be some synchronism between the vibrations caused in air or ether by the latter explosive, and the natural period of vibration of a gun-cotton molecule. At all events, the supposition is made that fulminate of mercury when exploded can produce vibrations which are not produced by explosions of nitro-glycerine ; and that the superior detonating power of fulminate of mercury may be due to the 
presence of these vibrations. The first set of experiments bearing on this point have been already discussed, with the result that the hypothesis is perhaps unnecessary. There are, however, a great number of other experiments, some of which cannot be so easily explained. In one case an explosion was induced, in a charge of fulminate of silver placed at the end of a tube, by the explosion of a similar charge at the other end. This effect was not interfered with by placing diaphragms across the tube; but the state of the internal surface of the tube seemed to exercise considerable influence. Experiments were also made on the action of fulminate of mercury on gun-cotton throngh tubes (Proc. Roy. Soc. 1874, vol. xxii. p. 160). The great influence exerted on the detonating power by the smoothness or roughness of the walls of the tube seems a strong argument against the supposed synchronism having much to do with the effect in these cases. On the other hand, it is just what we should expect if there was a bodily motion of air down the tube, or even if, as in the case where diaphragms were inserted, the motion was transmitted from layer to layer without any great amount of displacement in each individual particle. It seems possible that some of the vortex motion caused by the "break-down" might be transmitted through the tube, and that the diaphragms merely served to change the portions of air of which the rings were actually composed. I admit that this is not very satisfactory; but if the roughening of the internal surface of the tubes actually exerted the effect attributed to it, we are, I think, justified in supposing that the explosions were not caused by the transmission of vibrations through the material of the pipe itself. Again, vibrations, to be of any effect in producing chemical change, must be comparable, as to period, with the molecular vibrations. If such vibrations are transmitted through ether, it is difficult to see where the influence of chalking the inside of the tubes can come in, and if through air, their wave-length would be too small (as will be shown) to be likely to be much influenced by particles of the size of chalk-dust.

The similar experiments of Campion and Pellet (Comptes Rendus, lxxv.) are sufficiently explained by their statement that they used iodide of nitrogen. Unless any one likes to suppose that the period of a fiddle-string may be comparable with the period of an iodide of nitrogen molecule, the further experiments of Campion and Pellet cannot be held to have much bearing on the subject. One can only wonder that they found a string that would vibrate slowly enough not to fire their iodide. As to their experiments with mirrors, black- 
ened or otherwise, the results obtained might be anticipated on almost any theory except that of "synchronous vibration." For the vibrations supposed on this theory to be most active, would be precisely those absorbable by lampblack. This point has been investigated by Berthelot, in volume $i$. of the Treatise, in a manner which leaves little doubt that he misunderstood Abel's theory. In order to show the importance of vibrations in producing chemical change, Berthelot experimented on various chemicals by swinging them on tuningforks. No effect was produced, nor indeed was it to be expected, unless the reagents were of such a nature that they required intense shaking to keep them mixed. Berthelot also experimented on ozone at much higher frequencies of vibration by causing a tube filled with the gas, mixed with oxygen, to be set into violent longitudinal vibration. No change in the ordinary rate of decomposition of ozone was observed. This is very interesting, but does not, as far as I can see, tonch Abel's theory. In order to disprove the theory, Berthelot ought to have made his tube vibrate till it got luminous, and observed the effect on the ozone all the way up.

There are many well-established cases of torpedoes exploding one another by influence, and the same thing occurs in firing dynamite shots in mines. The former alone possess any interest for our present purpose. If the effects due to fulminate of mercury when fired under water are in any way similar to those which may be supposed to take place on the detonation of large charges of gun-cotton, then, by the experiments described above, it would be likely that quite extraordinary effects might be propagated in some cases. There ought, however, to be a capriciousness in the observed action of torpedoes on one another; but whether this has been observed or not, I am unfortunately unable to state. However, I will assume that it has not, and that here we have a case where the effect is largely due to "synchronous vibration." We will therefore consider the ways in which vibrations of sufficiently small period could be transmitted. I assume that no vibrations can have any influence unless they are of such a period as to be comparable with the natural period of vibration of the molecules of the substance to be xploded.

Let a body be gradually heated and its temperature measured as soon as light comes from it having the same refrangibility as the line $\mathrm{A}$ in the solar spectrum. Let the temperature be, say, of the order of $1500^{\circ}$ Centigrade. Then the molecules of the body will be vibrating in some way comparable with the period of the A line; that is, about $4 \times 10^{14}$ times per second. 
Suppose gun-cotton could be heated red-hot without decomposition, then its molecular period would be of this order. We are quite unable to say how the period varies with the temperature in solid bodies at low temperatures. But the spectroscope shows that it does not change much at high temperatures.

The only possible way of obtaining an idea would be to extend the spectroscopic investigation even further than it has been done by Abney; either photographically, or by means of a thermopile. We will assume, however, that as the bodies cool, their molecular vibrations, if altering at all as to period, tend to become slower, as well as of smaller amplitude. Let us consider the limiting condition of propagation of waves of longitudinal displacement. There seems no reason for supposing that the velocity of propagation would fall off till we come to waves of wave-length comparable with molecular distances; for instance, with the mean free path. Now by experiments on diffusion it seems that the mean free path in oxygen is of the order of $5.6 \times 10^{-6}$ centimetres; in sugar solution it is $10^{-5}$ of this, or $5 \cdot 6 \times 10^{-11}$ centimetres ; while in solids it is probably much less. The size of a molecule, however, seems to be of the order $5.8 \times 10^{-8}$ centim., so this will give our superior limit in liquids and solids.

Suppose that the smallest possible wave-length is the diameter of a molecule, and that the velocity of propagation is the same as that of sound, down to this limit. Then if V be the velocity of propagation, or the number of vibrations per second, and $\lambda$ the wave-length in water, we have

$$
n=\frac{V}{\lambda}=\frac{1 \cdot 4 \times 10^{5}}{5 \cdot 8 \times 10^{-8}}=2 \cdot 4 \times 10^{12} .
$$

But it is unlikely that we could get a wave-length anything like so small as this ; so let us take as our limiting value the wave-length equal to a thousand molecular diameters. This gives us for the limiting frequency

$$
n=2 \cdot 4 \times 10^{9} \text {. }
$$

Comparing this with the $n$ for the A line, which is $4 \times 10^{14}$, we see that it is about a million times too slow to produce any effect on molecules vibrating so as to emit red light. But bodies at the ordinary temperature might possibly vibrate slowly enough to be influenced directly, though this is unlikely. I must confess to being rather surprised that the numbers are as comparable as they seem to be. If we perform the same operation for gases, putting $\lambda=1000$ mean 
free paths, we get for oxygen $n=5 \times 10^{6}$. Here the discrepancy is a thousand times as great; so that if longitudinal vibrations are to be considered as likely to produce any effect, they will certainly be considerably more likely to do so if transmitted through solids or liquids than through gases.

We have still, however, got the ether to fall back on, and there we are safe, for there is no reason why vibration of the right period should not be transmitted through it.

The experiments with tubes, however, seem to point exclusively to the air as the medium through which vibrations are to be transmitted, and that may, I fancy, be fuirly regarded as unlikely. If the theory of synchronous vibration can be disproved at all by experiment, then Abel has, at all events, made the most telling experiment against it; there may of course be other experiments with which I am unacquainted, though not for want of endeavour on my part to discover them; and these may point in the opposite direction. Still, in the light of what has been published on the subject, there is little doubt that our natural hesitation to accept a theory of vibrations is justified by a consideration of the fucts. On the other hand, if we allow that vortex motion may exist, it will account for some of the effects observed in the neighbourhood of violent explosions. The most important effect to be accounted for is the capriciousness of explosions. Instances are so numerous that it is hardly worth while to dwell on them in detail ; I happen, however, to have an account of the famous explosion at Bremer Haven from an eye-witness, and was especially struck by the way in which the bystanders seemed actually selected for injury, and that not always from flying débris. Such effects as these are difficult to account for, on any theory of uniform propagation of wave-motion. Again, any of the observed phenomena of propagation of explosion are as well explained by vortex propagation as by wave-motion. There is no reason why the two states of propagation should not exist together, varying in their relative importance according as the explosion is of long or short duration. In ordinary cases of detonation I imagine the shocks are given chiefly by the explosion-gases before any considerable break-down has taken place. In conclusion, I wish to say that, though I have given prominence to the vortex-ring method of propagation, I do not intend to offer it as necessarily the most important of the phenomena in all cases, but only to point out that in some circumstances, chiefly those which have been inadequately explained, a consideration of its possible influence helps us to understand the facts. 